\title{
Absorption Spectra of Blue-Light-Emitting Oligoquinolines from Time-Dependent Density Functional Theory
}

\author{
Jianmin Tao, ${ }^{* \dagger \dagger}$ Sergei Tretiak, ${ }^{\dagger, \ddagger}$ and Jian-Xin $\mathrm{Zhu}^{\dagger}$ \\ Theoretical Division and Center for Nonlinear Studies, Los Alamos National Laboratory, Los Alamos, \\ New Mexico 87545, and Center for Integrated Nanotechnology, Los Alamos National Laboratory, \\ Los Alamos, New Mexico 87545
}

Received: May 27, 2008; Revised Manuscript Received: August 07, 2008

\begin{abstract}
Recently, it has been discovered that a series of four conjugated oligomers, oligoquinolines, exhibits many desirable properties of organic materials for developing high-performance light-emitting diodes: good blue color purity, high brightness, high efficiency, and high glass-transition temperatures. In this work, we investigate the optical absorption of oligoquinolines in the gas phase and chloroform $\left(\mathrm{CHCl}_{3}\right)$ solution, respectively, using time-dependent density functional theory with the adiabatic approximation for the dynamical exchange-correlation potential. Our calculations show that the first peak of optical absorption corresponds to the lowest singlet excited state, whereas several quasi-degenerate excited states contribute to the experimentally observed higher-frequency peak. We find that, compared with the gas phase, there is a moderate red shift in excitation energy in solution due to the solute-solvent interaction simulated using the polarizable continuum model. Our results show that the lowest singlet excitation energies of oligoquinolines in chloroform solution calculated with the adiabatic hybrid functional PBE0 are in a good agreement with experiments. Our simulated optical absorption agrees well with the experimental data. Finally, analysis of the natural transition orbitals corresponding to the excited states in question underscores the underlying electronic delocalization properties.
\end{abstract}

\section{Introduction}

Development of high-performance organic light-emitting diodes (OLEDs) ${ }^{1}$ plays a crucial role in the fabrication of highresolution, full-color, and flat-panel displays. ${ }^{2}$ The advantages of the OLEDs over the conventional inorganic semiconductor materials such as silicon and germanium are their ultralow cost, their light weight, and their flexibility. Furthermore, because of the ability to deposit organic films on any low-cost substrates $^{2-6}$ such as glass, plastic, or metal foils, OLED materials are particularly well suited for large-area displays. ${ }^{3}$ Successful commercial production of organic electrophotographic imaging ${ }^{7-9}$ for copiers, printers, and projection TV benefits from the improvement of material properties and optimization of device structure for OLEDs to enhance brightness, power efficiency, luminescence efficiency, and color purity of the three primary electroluminescence (EL) colors (red, green, and blue).

Ideal organic EL materials ${ }^{6}$ should be (i) readily processible, (ii) thermally stable (to withstand inevitable Joule heating generated during OLED operation), and (iii) simultaneously have high charge conductivity ${ }^{10-12}$ and high luminescence efficiency. However, the design and synthesis of such light-emitting organic materials with satisfactory multifunctional properties for highperformance OLEDs remain challenging. Recently, a series of four promising n-type (electron transport) blue-light-emitting $\pi$-conjugated oligomers (see Figure 1 ) have been synthesized. ${ }^{1}$ These oligoquinolines, 6,6-bis(2,4-diphenylquinoline) (B1PPQ), 6,6-bis(2-(4-tert-butylphenyl)-4-phenylquinoline) (BtBPQ), 6,6-

\footnotetext{
* To whom correspondence should be addressed.

$\dagger$ Theoretical Division and Center for Nonlinear Studies.

$\ddagger$ Center for Integrated Nanotechnology.
}
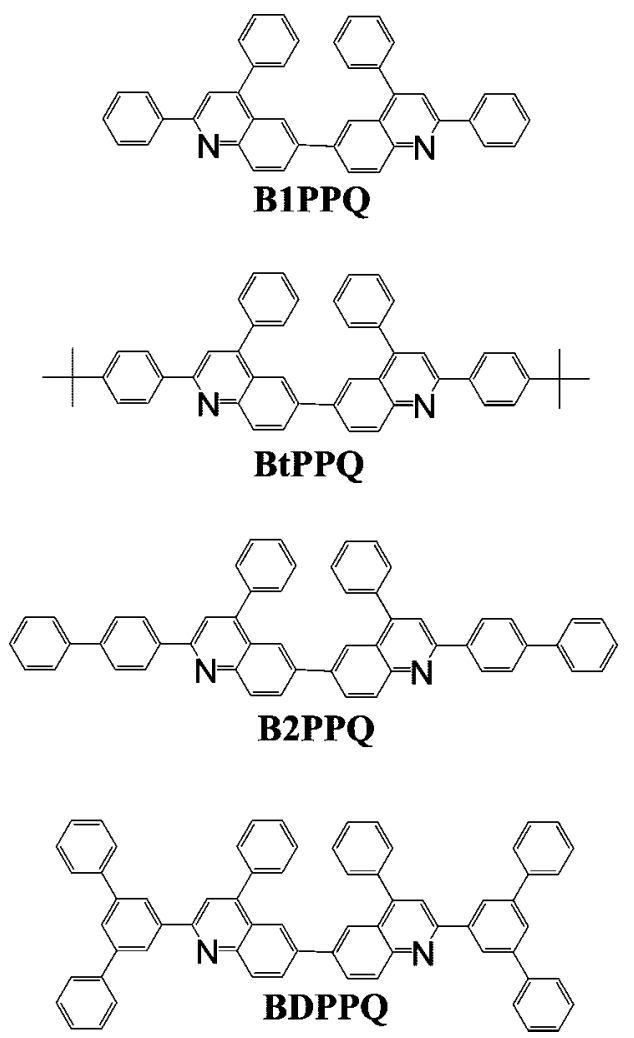

Figure 1. Molecular structures of the computationally studied bluelight-emitting oligoquinolines.

bis(2-p-biphenyl)-4-phenylquinoline) (B2PPQ), and 6,6-bis((3,5diphenylbenzene)-4-phenylquinoline) (BDBPQ), may have a 
potential for use in high-efficiency light-emitting diodes. Their optical properties such as absorption and emission spectra have been experimentally measured as well. ${ }^{1}$ Using these data, we put to the test several electronic structure methods based on the time-dependent extension of DFT for excited-state properties.

Such theoretical investigation of dynamical properties based on ab initio methods can lead to a better understanding of molecular electronic excited states, which is significantly important in the study of spectroscopy and photochemistry. In particular, it provides a relevant guide for the design and improvement of optical materials. The method of choice for the calculation of dynamical properties such as excitation energies is time-dependent density functional theory (TDDFT), ${ }^{13,14}$ which is the most important extension of the static DFT. It borrows the Kohn-Sham mapping theorem (or Kohn-Sham strategy), ${ }^{15}$ the central idea of the static DFT, and maps the complicated problem of interacting particles in a time-dependent external potential to a simpler one of noninteracting particles moving in a time-dependent effective potential. Since the formal proof of Runge and Gross, ${ }^{16}$ TDDFT has rapidly grown up to be one of the most popular electronic structure theories. No method can achieve the comparable accuracy of this theory with the same computational efforts.

The physics for the calculation of vertical excitation energies within the TDDFT formalism is simple. According to TDDFT, the induced density $n_{1}(\mathbf{r}, t)$ arising from the density response of the interacting system to an external perturbation is the same as that arising from the density response of the noninteracting system to the effective perturbation, which is the sum of the external perturbation, the induced Hartree $(\mathrm{H})$ and exchange-correlation (xc) potentials, $v_{\mathrm{s}, 1}(\mathbf{r}, t)=v_{1}(\mathbf{r}, t)+u_{\mathrm{H}, 1}(\mathbf{r}, t)+v_{\mathrm{xc}, 1}(\mathbf{r}, t)$. Making use of the key observation ${ }^{17}$ that the poles of the interacting density response are not equal to those of the noninteracting density response, we arrive from the linear response theory at an equation, from which the physical excitation energies can be obtained by solving an eigenvalue problem. The theory has been briefly outlined in ref 18 and described in detail in refs 17 and 19. An alternative treatment of this problem based on density matrices can be found in ref 20 .

In TDDFT, everything is known except the dynamical XC potential, which must be approximated as a functional of the instantaneous density $n(\mathbf{r}, t)$. The simplest construction is the adiabatic approximation, ${ }^{21}$ which makes use of the ground-state $\mathrm{XC}$ potential but replaces the ground-state density $n_{0}(\mathbf{r})$ with the instantaneous density $n(\mathbf{r}, t)$, namely

$$
v_{\mathrm{xc}}^{\mathrm{ad}}([n] ; \mathbf{r}, t)=\left.\frac{\delta E_{\mathrm{xc}}\left[n_{0}\right]}{\delta n_{0}(\mathbf{r})}\right|_{n_{0}(\mathbf{r})=n(\mathbf{r}, t)}
$$

It is known that the adiabatic approximation is adequate for the description of low-lying vertical excitations ${ }^{19}$ of molecules, for which the single pole approximation is valid, while it is not well justified for the description of multiparticle excitations due to the disregard of the frequency dependence ${ }^{22}$ arising from the vector potential. Although the nonadiabatic TDDFT ${ }^{23-27}$ takes into account the frequency-dependent vector potential, it requires much more effort (both on the theoretical and computational sides). As a result, the nonadiabatic part of the dynamical potential is usually ignored in practical applications. The adiabatic approximation has been widely used to describe lowlying excitations ${ }^{28-37}$ of molecules, in which single-particle excitations dominate.

Earlier tests show that the adiabatic XC potentials constructed from a ladder of commonly used nonempirical density func- tionals, the local spin density approximation (LSDA), the generalized gradient approximation (GGA) of Perdew, Burke, and Ernzerhof (PBE), ${ }^{38}$ and the meta-GGA of Tao, Perdew, Staroverov, and Scuseria (TPSS), ${ }^{39,40}$ yield excitation energies of small molecules in fairly good agreement with experiments, although these approximations were originally constructed for use in the static DFT, in which these semilocal functionals, in particular, the TPSS meta-GGA, can achieve consistent useful accuracy for molecules ${ }^{41-43}$ and solids ${ }^{44}$ and have been extended $^{45}$ to deal with systems in the presence of a magnetic field and employed to calculate the quantum stress tensor. ${ }^{46}$ Because transitions usually occur between high-occupied and low-unoccupied orbitals, the improvement of the XC potential in the outermost valence region or density tail region may result in the improvement of the description of low-lying excitations. This has been confirmed by the one-parameter hybrid functionals, $\mathrm{PBE0}^{30}$ (a hybrid of PBE with $25 \%$ exact exchange) and $\mathrm{TPSSh}^{41}$ (a hybrid of TPSS with $10 \%$ exact exchange). Both hybrids yield excitation energies consistently in better agreement ${ }^{18}$ with experiments than their parental nonhybrid functionals PBE GGA and TPSS meta-GGA. Furthermore, because PBE0 mixes more exact exchange than TPSSh, it is expected to give a better performance in the prediction of low-lying excitations.

In this work, the optical absorption of the four oligoquinolines is calculated using the adiabatic TDDFT. Here, we focus on the adiabatic PBE0, which yields the most accurate excitation energies of atoms and small molecules, ${ }^{18}$ while the results obtained with LSDA, TPSS meta-GGA, the TPSS-based oneparameter hybrid TPSSh, and the three-parameter hybrid B3LYP ${ }^{47}$ with $1 / 5$ exact exchange are also presented. Our calculations show that the first peak of optical absorption corresponds to the lowest singlet excited state, whereas several excited states that are degenerate or nearly degenerate contribute to the experimentally observed higher-frequency peak. We find that the lowest excitation energies of oligoquinolines in chloroform $\left(\mathrm{CHCl}_{3}\right)$ solution calculated with the adiabatic hybrid functional PBE0 are in good agreement with experiments. We also calculate the oscillator strengths and dipole moments of the oligoquinoline molecules both in the gas phase and in chloroform solution. We show that both oscillator strength and dipole moment are larger in solution than in the gas phase, as expected. These two quantities are directly related to the peak magnitude or absorption intensity in the UV/visible absorption spectra. By comparing the simulated absorption spectra in the gas phase with those in chloroform solution, we find that, relative to the excitation energy in the gas phase, there is a consistent red shift in the excitation energy in solution due to the solute-solvent interaction. Finally, analysis of the natural transition orbitals ${ }^{48}$ corresponding to the excited states in question is made to spell out the underlying electronic delocalization properties.

\section{Computational Method}

All calculations are performed using the GAUSSIAN 03 suite. ${ }^{49}$ First, we optimize the ground-state geometries of oligoquinolines by performing the self-consistent ground-state calculation with respective density functionals. Then, we evaluate the vertical excitation energies of these oligomers based on the optimized ground-state geometries with the respective adiabatic functionals. For consistency, the same basis set 6-31G(2df,p) was used in all calculations. While this basis set is not as large as the one we used in previous calculations, ${ }^{18}$ the discrepancy arising from this basis set difference is 
TABLE 1: B1PPQ: Singlet and Triplet Vertical Excitation Energies $\left(\omega_{\mathrm{S}}^{n}, \omega_{\Gamma}^{n}, n=\right.$ the $n$th excited state) in eV, the Transition Oscillator Strength $\left(f^{\mathrm{abs}, n}\right)$, and the Dipole Moment of the Ground State in Debye of the B1PPQ Molecule in the Gas Phase $\left(\mu_{\mathrm{g}}\right)$ and in Chloroform Solution $\left(\mu_{\text {sol }}\right)$, Calculated Using the Five Adiabatic Density Functionals with the Basis Set 6-31G(2df,p) and the Geometry Optimized on the Respective Density Functionals with the Same Basis ${ }^{a}$

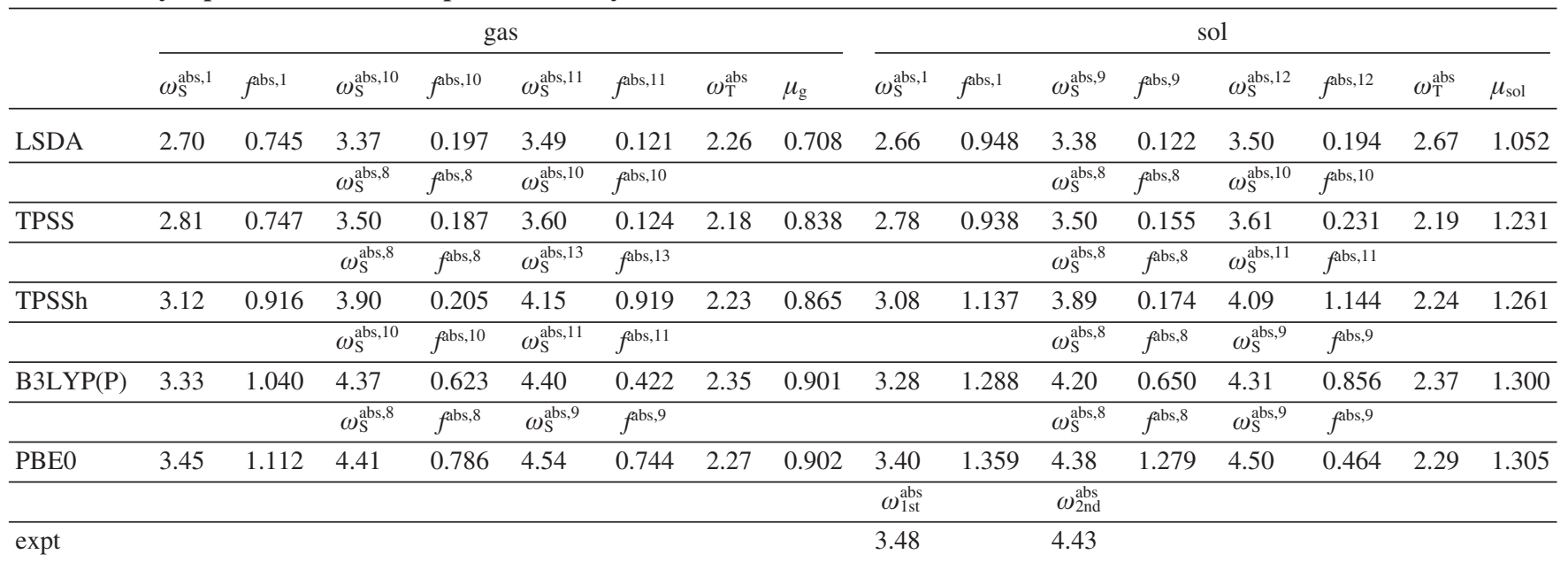

${ }^{a} 1 \mathrm{eV}=8065.5 \mathrm{~cm}^{-1}=0.03675$ hartree. The energy (in units of eV) of the wavelength $\lambda$ (in units of $\mathrm{nm}$ ) is $h c / \lambda=(\mathrm{nm} / \lambda$ ) $1239.84 \mathrm{eV}$, where $h$ is Planck's constant and $c$ is the speed of light. Experimental values measured in chloroform are obtained from ref 1 .

TABLE 2: BtBPQ: Singlet and Triplet Vertical Excitation Energies $\left(\omega_{\mathrm{S}}^{n}, \omega_{\mathrm{T}}^{n}, n=\right.$ the $n$th excited state), the Transition Oscillator Strength $\left(f^{\mathrm{abs}, n}\right)$, and the Dipole Moment of the Ground State in Debye of the BtBPQ Molecule in the Gas Phase $\left(\mu_{\mathrm{g}}\right)$ and in Chloroform Solution $\left(\mu_{\text {sol }}\right)$, Calculated Using the Five Adiabatic Density Functionals with the Basis Set 6-31G(2df,p) and the Geometry Optimized on the Respective Density Functionals with the Same Basis ${ }^{a}$

\begin{tabular}{|c|c|c|c|c|c|c|c|c|c|c|c|c|c|c|c|c|}
\hline & \multicolumn{8}{|c|}{ gas } & \multicolumn{8}{|c|}{ sol } \\
\hline \multirow[t]{2}{*}{ LSDA } & 2.64 & 0.896 & 3.33 & 0.316 & 3.61 & 0.3685 & 2.24 & 0.699 & 2.61 & 1.095 & 3.34 & 0.535 & 3.59 & 0.485 & 2.25 & 1.010 \\
\hline & & & $\omega_{\mathrm{S}}^{\mathrm{abs}, 8}$ & $f^{\mathrm{abs}, 8}$ & $\omega_{\mathrm{S}}^{\mathrm{abs}, 15}$ & $f^{\mathrm{abs}, 15}$ & & & & & $\omega_{\mathrm{S}}^{\mathrm{abs}, 8}$ & $f^{\mathrm{abs}, 8}$ & $\omega_{\mathrm{S}}^{\mathrm{abs}, 13}$ & $f^{\mathrm{abs}, 13}$ & & \\
\hline \multirow[t]{2}{*}{ TPSS } & 2.77 & 0.868 & 3.47 & 0.458 & 3.73 & 0.339 & 2.18 & 0.844 & 2.74 & 1.058 & 3.47 & 0.536 & 3.69 & 0.207 & 2.19 & 1.220 \\
\hline & & & $\omega_{\mathrm{S}}^{\mathrm{abs}, 8}$ & $f^{\mathrm{abs}, 8}$ & $\omega_{\mathrm{S}}^{\mathrm{abs}, 13}$ & $f^{\mathrm{abs}, 13}$ & & & & & $\omega_{\mathrm{S}}^{\mathrm{abs}, 8}$ & $f^{\mathrm{abs}, 8}$ & $\omega_{\mathrm{S}}^{\mathrm{abs}, 11}$ & $f^{\mathrm{abs}, 11}$ & & \\
\hline TPSSh & & & $\omega_{\mathrm{S}}^{\mathrm{abs}, 8}$ & $f^{\mathrm{abs}, 8}$ & $\omega_{\mathrm{S}}^{\mathrm{abs}, 11}$ & $f^{\mathrm{abs}, 11}$ & & & & & $\omega_{\mathrm{S}}^{\mathrm{abs}, 8}$ & $f^{\mathrm{abs}, 8}$ & $\omega_{\mathrm{S}}^{\mathrm{abs}, 11}$ & $f^{\mathrm{abs}, 11}$ & & \\
\hline \multirow[t]{2}{*}{ B3LYP } & 3.29 & 1.255 & 4.17 & 0.764 & 4.36 & 0.753 & 2.35 & 0.916 & 3.25 & 1.473 & 4.16 & 0.931 & 4.32 & 0.704 & 2.36 & 1.300 \\
\hline & & & $\omega_{\mathrm{S}}^{\mathrm{abs}, 8}$ & $f^{\text {abs, } 8}$ & $\omega_{\mathrm{S}}^{\mathrm{abs}, 11}$ & $f^{\mathrm{abs}, 11}$ & & & & & $\omega_{\mathrm{S}}^{\mathrm{abs}, 8}$ & $f^{\mathrm{abs}, 8}$ & $\omega_{\mathrm{S}}^{\mathrm{abs}, 11}$ & $f^{\mathrm{abs}, 11}$ & & \\
\hline \multirow[t]{2}{*}{ BPE0 } & 3.41 & 1.346 & 4.36 & 1.160 & 4.56 & 0.415 & 2.27 & 0.909 & 3.37 & 1.575 & 4.33 & 1.431 & 4.53 & 0.299 & 2.28 & 1.286 \\
\hline & & & & & & & & & $\omega_{1 \mathrm{st}}^{\mathrm{abs}}$ & & $\omega_{2 \mathrm{nd}}^{\mathrm{abs}}$ & & & & & \\
\hline
\end{tabular}

${ }^{a}$ Experimental values measured in chloroform are obtained from ref 1.

negligibly small, and our conclusion based on the present basis set should not be changed. For a detailed comparison and discussion of basis set effects, see refs 50 and 51 . The tight self-consistent field convergence criteria $(\mathrm{SCF}=\mathrm{Tight})$ are used in all calculations.

Since the PBE and TPSS correlation functionals use the Perdew-Wang parametrization ${ }^{52}$ for the LSDA correlation energy as their local part, for consistency, this parametrization is used for all LSDA calculations. We further calculate the excitation energies and the dipole moments of the four organic oligomers in chloroform solvent using two methods, PCM (polarizable continuum model) ${ }^{53}$ and CPCM (conductor-like PCM) ${ }^{54,55}$ Both solvation models have been developed for the treatment of solute-solvent interactions in the calculations of energies, geometric structures, harmonic frequencies, and other electronic properties in solution. Our calculations show that both models give nearly the same results. Here, we only report the results calculated with the PCM method.

\section{Results and Discussion}

Tables $1-4$ show the summary of calculated selected excitedstate quantities of oligoquinolines (B1PPQ, BtBPQ, B2PPQ, BDBPQ; see Figure 1) in the gas phase and solution, respectively. We tabulate the excitation energies of three singlet states with the largest oscillator strengths in the UV/visible region, the corresponding oscillator strengths, the excitation energy of the first triplet state, and the value of the ground-state dipole moment in the gas phase and solution.

To simulate the experimentally observed absorption with our calculations (see Figures 2-5), a simple analytic expression for the normalized absorption intensity or peak magnitude is assumed as

$$
I(\omega)=\sum_{i} f\left(\omega_{i}\right) \delta_{m}\left(\omega-\omega_{i}\right) / \sum_{i} f\left(\omega_{i}\right)
$$


TABLE 3: B2PPQ: Singlet and Triplet Vertical Excitation Energies $\left(\omega_{\mathrm{S}}^{n}, \omega_{\Gamma}^{n}, n=\right.$ the $n$th excited state) in eV, the Transition Oscillator Strength $\left(f^{\mathrm{abs}, n}\right)$, and the Dipole Moment of the Ground State in Debye of the B2PPQ Molecule in the Gas Phase $\left(\mu_{\mathrm{g}}\right)$ and in Chloroform Solution $\left(\mu_{\text {sol }}\right)$, Calculated Using the Five Adiabatic Density Functionals with the Basis Set 6-31G(2df,p) and the Geometry Optimized on the Respective Density Functionals with the Same Basis ${ }^{a}$

\begin{tabular}{|c|c|c|c|c|c|c|c|c|c|c|c|c|c|c|c|c|}
\hline & \multicolumn{8}{|c|}{ gas } & \multicolumn{8}{|c|}{ sol } \\
\hline LSDA & & & $\omega_{\mathrm{S}}^{\mathrm{abs}, 5}$ & $f^{\mathrm{abs}, 5}$ & $\omega_{\mathrm{S}}^{\mathrm{abs}, 9}$ & $f^{\text {abs, } 9}$ & & & & & $\omega_{\mathrm{S}}^{\mathrm{abs}, 5}$ & $f^{\mathrm{abs}, 5}$ & $\omega_{\mathrm{S}}^{\mathrm{abs}, 15}$ & $f^{\mathrm{abs}, 15}$ & & \\
\hline \multirow[t]{2}{*}{ TPSS } & 2.62 & 1.135 & 3.19 & 0.366 & 3.35 & 0.261 & 2.11 & 0.828 & 2.59 & 1.332 & 3.17 & 0.590 & 3.62 & 0.193 & 2.12 & 1.227 \\
\hline & & & $\omega_{\mathrm{S}}^{\mathrm{abs}, 7}$ & $f^{\mathrm{abs}, 7}$ & $\omega_{\mathrm{S}}^{\mathrm{abs}, 13}$ & $f^{\mathrm{abs}, 13}$ & & & & & $\omega_{\mathrm{S}}^{\mathrm{abs}, 7}$ & $f^{\mathrm{abs}, 7}$ & $\omega_{\mathrm{S}}^{\mathrm{abs}, 13}$ & $f^{\mathrm{abs}, 13}$ & & \\
\hline TPSSh & & & $\omega_{\mathrm{S}}^{\mathrm{abs}, 8}$ & $f^{\mathrm{abs}, 8}$ & $\omega_{\mathrm{S}}^{\mathrm{abs}, 13}$ & $f^{\mathrm{abs}, 13}$ & & & & & $\omega_{\mathrm{S}}^{\mathrm{abs}, 6}$ & $f^{\mathrm{abs}, 6}$ & $\omega_{\mathrm{S}}^{\mathrm{abs}, 13}$ & $f^{\mathrm{abs}, 13}$ & & \\
\hline \multirow[t]{2}{*}{ B3LYP } & 3.18 & 1.690 & 4.01 & 0.692 & 4.32 & 0.876 & 2.31 & 0.908 & 3.14 & 1.952 & 3.98 & 0.851 & 4.29 & 0.885 & 2.32 & 1.308 \\
\hline & & & $\omega_{\mathrm{S}}^{\text {abs }, 8}$ & $f^{\mathrm{abs}, 8}$ & $\omega_{\mathrm{S}}^{\mathrm{abs}, 13}$ & $f^{\text {abs, } 13}$ & & & & & $\omega_{\mathrm{S}}^{\mathrm{abs}, 8}$ & $f^{\text {abs, } 8}$ & $\omega_{\mathrm{S}}^{\mathrm{abs}, 13}$ & $f^{\text {abs, } 13}$ & & \\
\hline \multirow[t]{2}{*}{ PBE0 } & 3.31 & 1.812 & 4.18 & 1.155 & 4.52 & 0.808 & 2.23 & 0.889 & 3.26 & 2.082 & 4.18 & 0.753 & 4.49 & 0.688 & 2.25 & 1.287 \\
\hline & & & & & & & & & $\omega_{1 \mathrm{st}}^{\mathrm{abs}}$ & & $\omega_{2 \mathrm{nd}}^{\mathrm{abs}}$ & & & & & \\
\hline
\end{tabular}

${ }^{a}$ Experimental values measured in chloroform are obtained from ref 1.

TABLE 4: BDBPQ: Singlet and Triplet Vertical Excitation Energies $\left(\omega_{\mathrm{S}}^{n}, \omega_{\mathrm{T}}^{n}, \boldsymbol{n}=\right.$ the $n$th excited state) in eV, the Transition Oscillator Strength $\left(f^{\mathrm{abs}, n}\right)$, and the Dipole Moment of the Ground State in Debye of the BDBPQ Molecule in the Gas Phase ( $\left.\mu_{\mathrm{g}}\right)$ and Chloroform Solution $\left(\mu_{\text {sol }}\right)$, Calculated Using the Five Adiabatic Density Functionals with the Basis Set 6-31G(2df,p) and the Geometry Optimized on the Respective Density Functionals with the Same Basis ${ }^{a}$

\begin{tabular}{|c|c|c|c|c|c|c|c|c|c|c|c|c|c|c|c|c|}
\hline & \multicolumn{8}{|c|}{ gas } & \multicolumn{8}{|c|}{ sol } \\
\hline \multirow[t]{2}{*}{ LSDA } & 2.63 & 0.683 & 2.70 & 0.173 & 3.26 & 0.149 & 2.25 & 0.772 & 2.61 & 0.896 & 2.70 & 0.140 & 3.31 & 0.131 & 2.26 & 1.092 \\
\hline & & & $\omega_{\mathrm{S}}^{\mathrm{abs}, 12}$ & $f^{\mathrm{abs}, 12}$ & $\omega_{\mathrm{S}}^{\mathrm{abs}, 14}$ & $f^{\mathrm{abs}, 14}$ & & & & & $\omega_{\mathrm{S}}^{\text {abs }, 10}$ & $f^{\text {abs, } 10}$ & $\omega_{\mathrm{S}}^{\mathrm{abs}, 14}$ & $f^{\text {abs, } 14}$ & & \\
\hline \multirow[t]{2}{*}{ TPSS } & 2.76 & 0.797 & 3.36 & 0.120 & 3.46 & 0.246 & 2.18 & 0.891 & 2.74 & 0.957 & 3.37 & 0.143 & 3.44 & 0.266 & 2.19 & 1.231 \\
\hline & & & $\omega_{\mathrm{S}}^{\mathrm{abs}, 13}$ & $f^{\mathrm{abs}, 13}$ & $\omega_{\mathrm{S}}^{\mathrm{abs}, 15}$ & $f^{\mathrm{abs}, 15}$ & & & & & $\omega_{\mathrm{S}}^{\mathrm{abs}, 6}$ & $f^{\mathrm{abs}, 6}$ & $\omega_{\mathrm{S}}^{\mathrm{abs}, 13}$ & $f^{\text {abs, } 13}$ & & \\
\hline TPSSh & & & $\omega_{\mathrm{S}}^{\mathrm{abs}, 12}$ & $f^{\mathrm{abs}, 12}$ & $\omega_{\mathrm{S}}^{\mathrm{abs}, 14}$ & $f^{\text {abs, } 14}$ & & & & & $\omega_{\mathrm{S}}^{\mathrm{abs}, 5}$ & $f^{\mathrm{abs}, 5}$ & $\omega_{\mathrm{S}}^{\mathrm{abs}, 12}$ & $f^{\mathrm{abbs}, 12}$ & & \\
\hline \multirow[t]{2}{*}{ B3LYP } & 3.31 & 1.227 & 4.14 & 0.446 & 4.27 & 0.153 & 2.36 & 1.014 & 3.28 & 1.400 & 3.83 & 0.171 & 4.13 & 0.436 & 2.37 & 1.361 \\
\hline & & & $\omega_{\mathrm{S}}^{\mathrm{abs}, 10}$ & $f^{\mathrm{abs}, 10}$ & $\omega_{\mathrm{S}}^{\mathrm{abs}, 13}$ & $f^{\mathrm{abs}, 13}$ & & & & & $\omega_{\mathrm{S}}^{\mathrm{abs}, 10}$ & $f^{\mathrm{abs}, 10}$ & $\omega_{\mathrm{S}}^{\mathrm{abs}, 13}$ & $f^{\text {abs, } 13}$ & & \\
\hline \multirow[t]{2}{*}{ PBE0 } & 3.42 & 1.321 & 4.31 & 0.644 & 4.45 & 0.270 & 2.27 & 0.982 & 3.39 & 1.501 & 4.29 & 0.842 & 4.44 & 0.407 & 2.28 & 1.335 \\
\hline & & & & & & & & & $\omega_{1 \mathrm{st}}^{\mathrm{abs}}$ & & $\omega_{2 \mathrm{nd}}^{\mathrm{abs}}$ & & & & & \\
\hline
\end{tabular}

${ }^{a}$ Experimental values measured in chloroform are obtained from ref 1.

where $\delta_{\mathrm{m}}(x)$ is a $\delta$-like function defined by

$$
\delta_{m}(x)=\frac{m}{\pi} \frac{1}{1+m^{2} x^{2}}
$$

with the properties ${ }^{56}$ of

$$
\int_{-\infty}^{\infty} \mathrm{d} x \delta_{m}(x)=1
$$

and

$$
\lim _{m \rightarrow \infty} \delta_{m}(x) \rightarrow \delta(x)
$$

Here, $m=15.5$ is determined by a fit to experiments and is valid for the four oligoquinoline molecules in the gas phase and solution. "Normalized absorption intensity" here means that that is, the area under the curve of $I(\omega)$ (see Figures 2-5) is 1.

$$
\int_{0}^{\infty} I(\omega) \mathrm{d} \omega=1
$$

Calculated electronic states are further studied using the natural transition orbital analysis of the excited states ${ }^{48}$ based on the calculated transition density matrices. This analysis offers the most compact representation of a given transition density in terms of an expansion into single-particle transitions.

Since the PBE0 method generally provides the best quantitative results in comparison with experimental data, we use this method to simulate the absorption spectra (Figures 2-5) and analyze the respective transition orbitals (Tables 5-8).

A. B1PPQ and BtBPQ. The calculated energies of the lowest excited state of B1PPQ in the gas phase listed in Table 


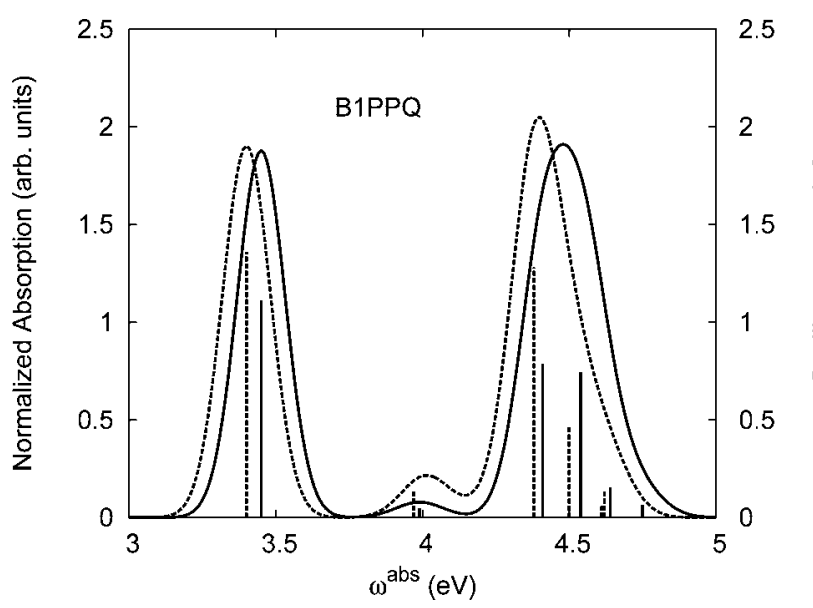

Figure 2. Normalized absorption $I$ of eq 2 (in arbitrary units) (right side) and oscillator strength $f$ (left side) of B1PPQ. The solid and dashed curves represent the normalized absorption in the gas phase and solution, while the solid and dashed "sticks" represent the oscillator strength in the gas phase and solution, respectively. The absorption wavelength $\lambda^{\text {abs }}$ (in units of $\mathrm{nm}$ ) may be obtained from the relation $\lambda^{\text {abs }}=(1239.84$ $\left.\mathrm{eV} / \omega^{\mathrm{abs}}\right)(\mathrm{nm})$, where $\omega^{\text {abs }}$ is the absorption frequency (in units of $\mathrm{eV}$ ).

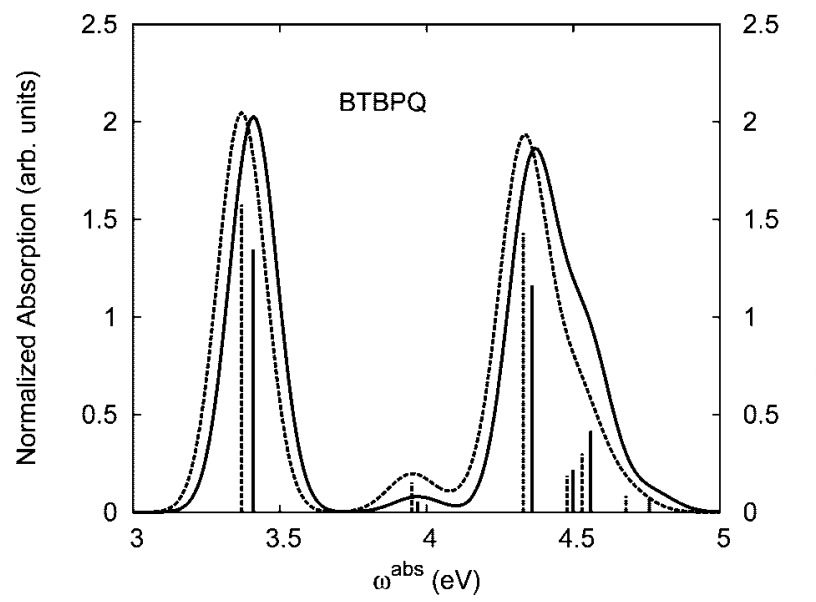

Figure 3. Normalized absorption $I$ of eq 2 (in arbitrary units) (right side) and oscillator strength $f$ (left side) of BtBPQ. The solid and dashed curves represent the normalized absorption in the gas phase and solution, while the solid and dashed "sticks" represent the oscillator strength in the gas phase and solution, respectively.

1 show a pronounced blue shift along the density functional models used from LSDA to meta-GGA to hybrid functionals. We observe a strong sensitivity to the fraction of the exact orbital exchange used in the functional. The total blue shift when going from LSDA to PBE0 (functional with $25 \%$ portion of the exact orbital exchange) is about $0.8 \mathrm{eV}$. Due to the nearly nonpolar structure of the molecule, we observe a fairly small solvatochromic shift of about $50 \mathrm{meV}$. The calculated PBE0 value for the excitation energy in chloroform, $3.40 \mathrm{eV}$, agrees well with the experimental maximum $(3.48 \mathrm{eV})$ of the lowest absorption peak. We note that such comparisons can be done only approximately since vibrational progression and disorder effects are lacking in our calculations. Such phenomena can account for up to a $0.1-0.2 \mathrm{eV}$ difference. ${ }^{57}$

The lowest excited state of B1PPQ has a sizable oscillator strength. Due to Kasha's rule, ${ }^{58}$ this state is also responsible for molecular luminescence, where the oscillator strength defines an efficiency of this process. The calculated oscillator strength tends to increase with the increase of the fraction of the exact orbital exchange in the functional. We observe about a $50 \%$

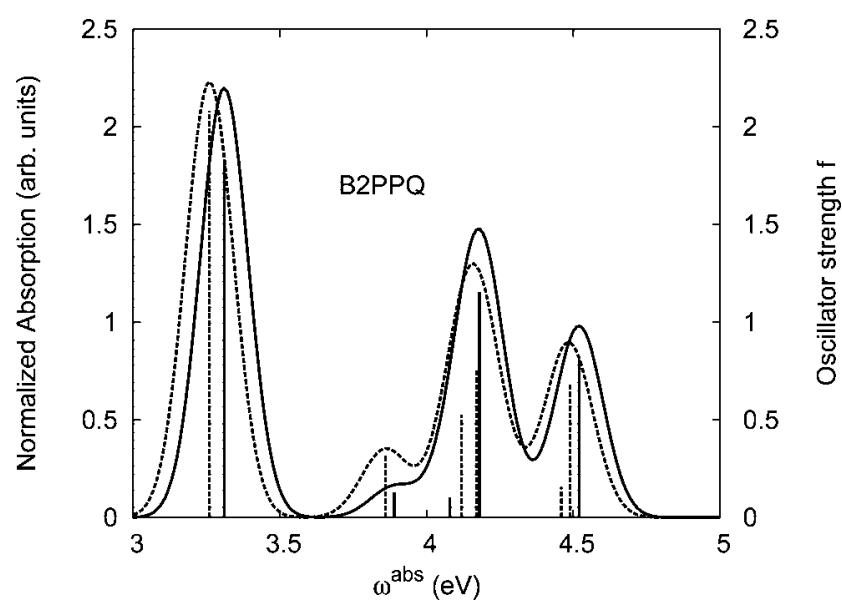

Figure 4. Normalized absorption $I$ of eq 2 (in arbitrary units) (right side) and oscillator strength $f$ (left side) of $\mathrm{B} 2 \mathrm{PPQ}$. The solid and dashed curves represent the normalized absorption in the gas phase and solution, while the solid and dashed "sticks" represent the oscillator strength in the gas phase and solution, respectively.

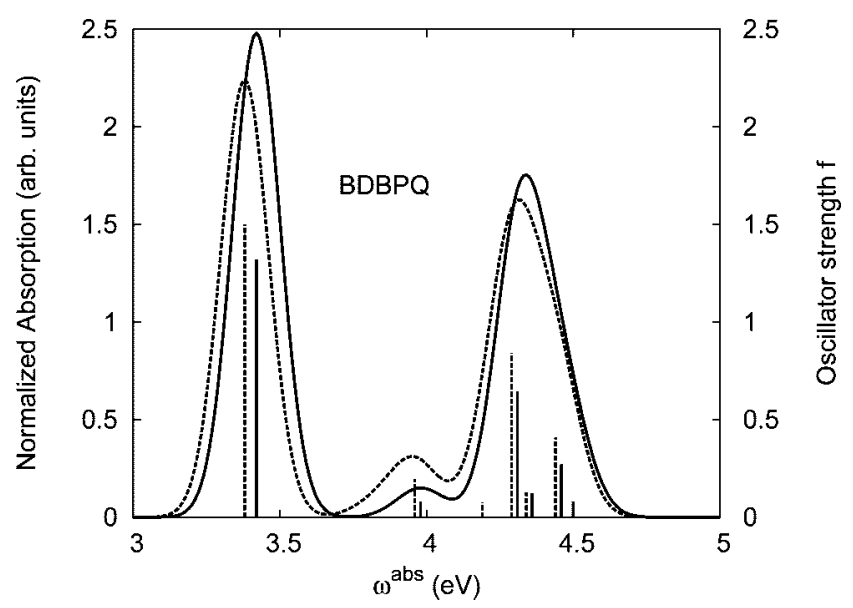

Figure 5. Normalized absorption $I$ of eq 2 (in arbitrary units) (right side) and oscillator strength $f$ (left side) of BDBPQ. The solid and dashed curves represent the normalized absorption in the gas phase and solution, while the solid and dashed "sticks" represent the oscillator strength in the gas phase and solution, respectively.

difference between computed LSDA and PBE0 values of the oscillator strength. In contrast to the energy values, solvent leads to a noticeable increase of the oscillator strength, compared to the gas phase values. Trends observed for calculated groundstate dipole moment values (see Table 1) are very similar to those for oscillator strengths. (The latter are directly relevant to the respective transition dipole moments from the ground state to the excited state.)

The experimental optical absorption ${ }^{1}$ of B1PPQ has a second peak appearing at $4.43 \mathrm{eV}$ (not shown here). Our calculations consistently predict that two excited states with nearly the same oscillator strength contribute to the intensity of this peak. These states are separated by about $0.1 \mathrm{eV}$ across all density functional models used. However, similar to the lowest state, the blue shift up to $1 \mathrm{eV}$ is observed when going along the line of density functional models used, from LSDA to PBE0. These two higherlying states have very similar solvatochromatic shifts as well. Again, PBE0 provides the most accurate transition energy values when compared to the experimental data. It is interesting to note that the oscillator strength of these two higher-lying states grows dramatically in the hybrid models. We observe a 4-6 fold increase when going from LSDA to PBE0. According to the LSDA (TPSS) results, the second peak magnitude should 
TABLE 5: TDDFT Natural Transition Orbital Analysis for the Three Excited States with the Largest Oscillator Strengths in B1PPQ in the Gas Phase ${ }^{a}$

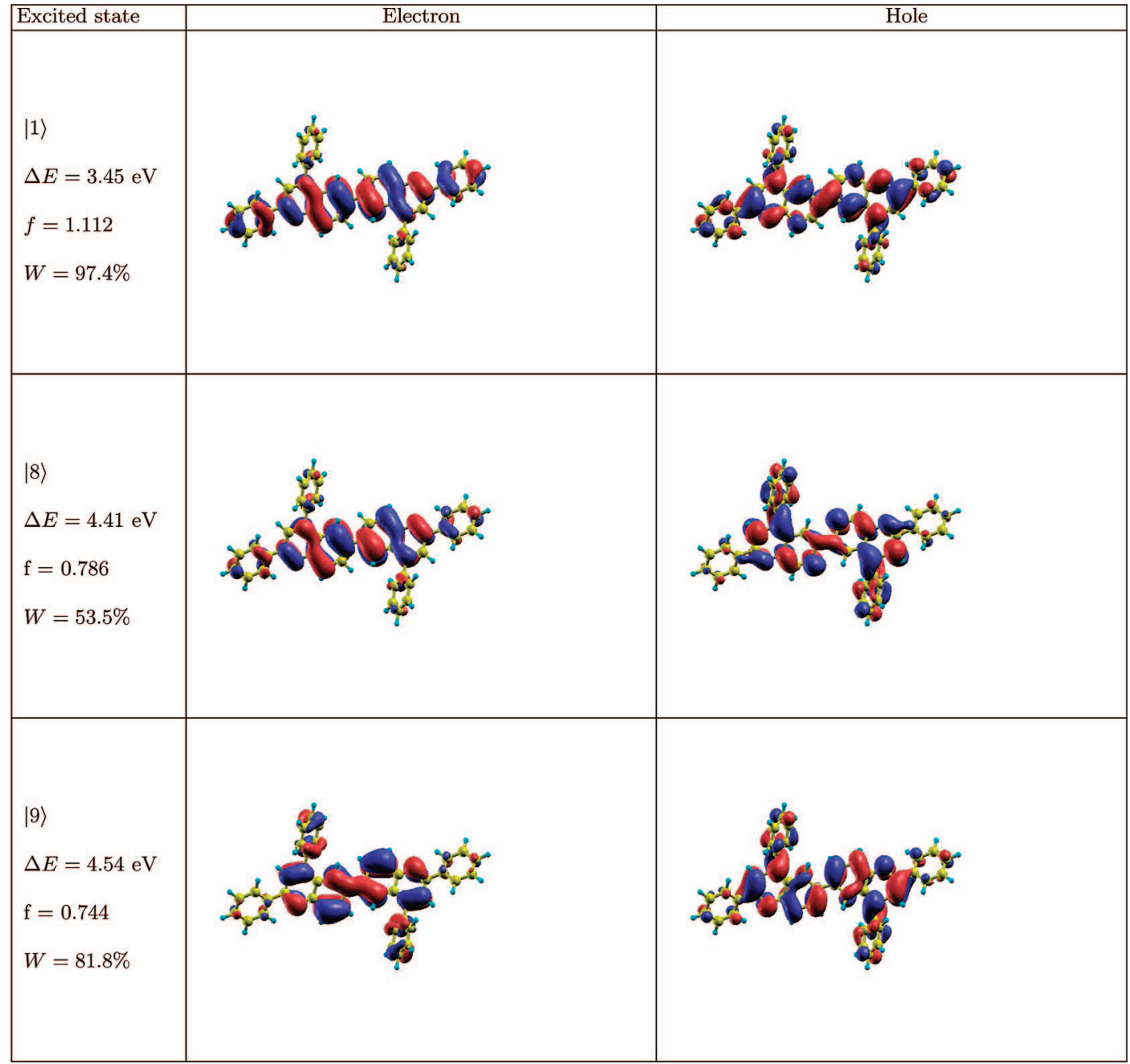

${ }^{a} \Delta E$ is the excitation energy, $f$ is the corresponding oscillator strength, and $W$ is the weight of the plotted orbital in the respective transition density matrix.

be much smaller compared to that of the first one. This is not, however, the case of the experiment, where the second peak has a larger amplitude compared to the first one. ${ }^{1}$ PBE0 nearly captures the experimental observations. Figure 2 displays the oscillator strength of B1PPQ as a function of absorption frequency $\omega$ (solid "stick" in the gas phase and dashed "stick" in solution) and our simulation of eq 2 for the normalized absorption band intensity as a function of $\omega$ (solid curve in the gas phase and dashed curve in solution) obtained from the PBE0 results. We note that the second peak has more intensity (integrated area under the curve) since it is composed from the two overlapping electronic transitions.

For reference, Table 1 lists calculated energies of the first triplet state. Triplet states are important for the efficiency of the lightemitting devices based on organic conjugated molecules. Their energetics and delocalization properties affect the dynamics of the charge recombination. ${ }^{59}$ Moreover, the lowest triplet state is responsible for weak phosphorescence in such systems. ${ }^{60}$ We note that the calculated energies of the first triplet state do not change substantially for all methods, which means that the singlet/triplet gap splitting grows significantly from nonhybrid to hybrid functionals, reaching $1.2 \mathrm{eV}$ for the PBE0 model. Such a theoretical prediction is likely close to the experimental case since such organic molecules generally exhibit large values of singlet/triplet gap due to low dimensionality and quantum confinement. ${ }^{60}$ As a rule of thumb, for many molecular systems, the first triplet state is lying approximately at $2 / 3$ of the value of the singlet gap, which is well predicted by the PBE0 model.

To analyze the electronic nature of calculated singlet excited states, we further utilize a natural transition orbital representation, as shown in Tables 5 and 6. We plot the orbitals derived from PBE0 computational results since this method provides the most accurate results in comparison with experiment across the entire molecular family considered. We first note that all considered excited states are $\pi-\pi^{*}$ excitations, as illustrated by their transition orbitals. The lowest excited state $|1\rangle$ can essentially be represented by a single pair of transition orbitals (see Table 5). This is a delocalized excitation involving the conjugated backbone of the B1PPQ oligomer. The side phenyl rings do not participate substantially in this optical excitation. Excited states $|8\rangle$ and $|9\rangle$ contributing to the second absorption peak are mainly delocalized in the middle section of the molecule. We notice that state $|8\rangle$ is multiconfigurational, that is, it can be represented by several dominating pairs of transition orbitals. Here, only the dominant contribution is shown in Table 5

BtBPQ has an identical conjugated molecular structure with B1PPQ. Methylated $\sigma$-bonded ends at BtBPQ do not introduce any substantial effects into the excited-state electronic structure. 
TABLE 6: TDDFT Natural Transition Orbital Analysis for the Three Excited States with the Largest Oscillator Strengths in BtBPQ in the Gas Phase ${ }^{a}$

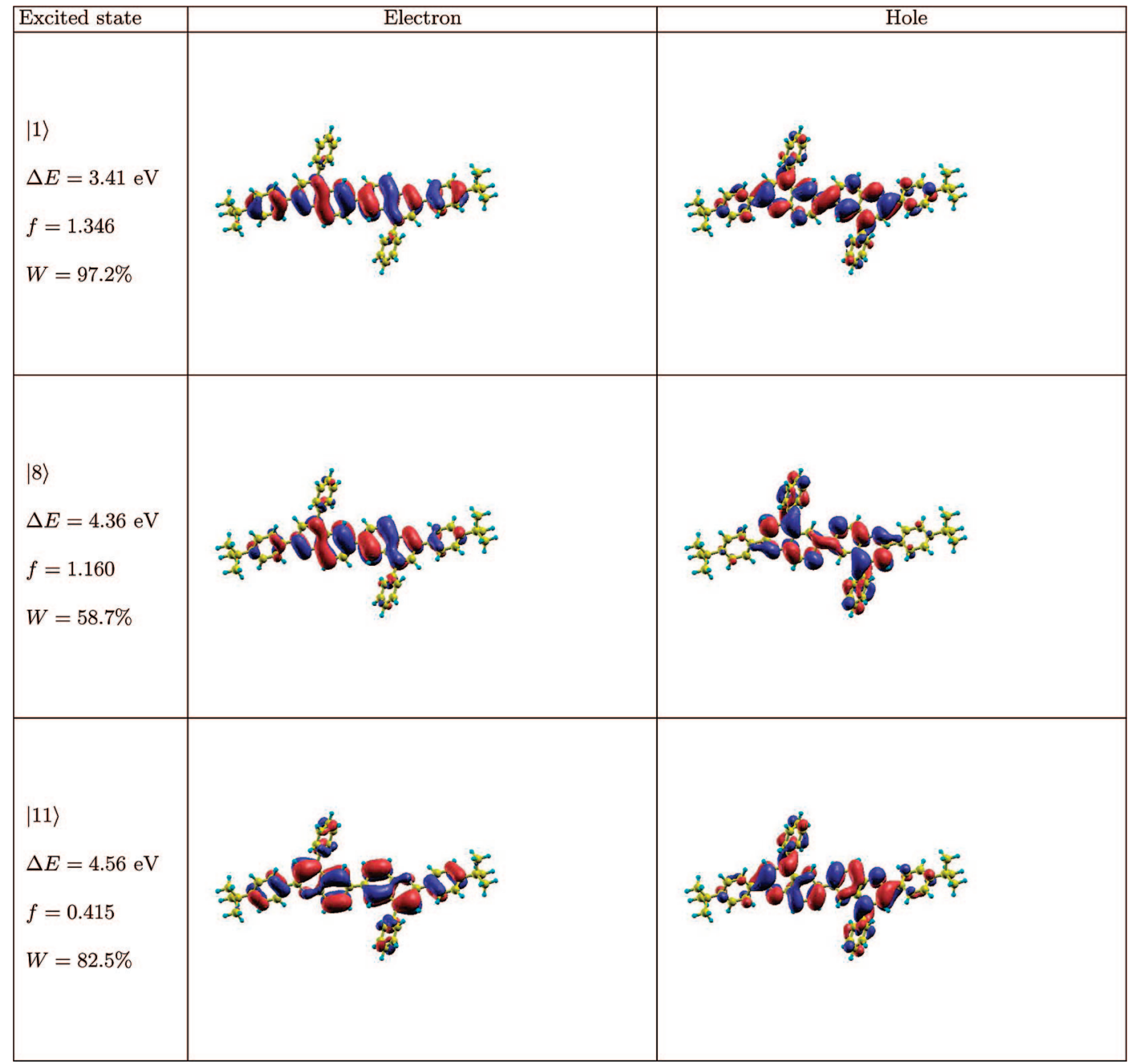

${ }^{a} \Delta E$ is the excitation energy, $f$ is the corresponding oscillator strength, and $W$ is the weight of the plotted orbital in the respective transition density matrix.

Nevertheless, these methyls yield consistent small red shifts of the first lowest (and dominant) singlet-singlet excitation energies of BtBPQ (on the order of several tenths of meV) across all computational results and experimental data compared to those of B1PPQ (see Tables 1 and 2). A slightly more noticeable effect is an increase of the oscillator strength of the lowest excited state, $|1\rangle$, in BtBPQ. This can be rationalized by examining the respective transition orbitals (Table 4), where the elongated molecular ends provide slightly larger room for electronic delocalization, which is reflected in the values of the dipole moments. Compared to B1PPQ, the intensity in the higher-energy absorption peak is shifted toward the lower state. Notably, both solvent effect and methylation (BtBPQ) lead to this effect (compare Figures 2 and 3). Finally, we emphasize that the slightly different chemical structure of B1PPQ and BtBPQ has no effect on the energies of their first triplet states.

B. B2PPQ and BDBPQ. Compared to B1PPQ, the molecular structure of B2PPQ has a longer conjugated backbone, whereas BDBPQ features four aryl substituents at meta-positions at both ends (see Figure 1). Even though the main physical phenomena and trends for B1PPQ discussed above are the same for B2PPQ and BDBPQ, here, we emphasize a few observed differences due to different molecular compositions.

The energy of the lowest singlet molecular state of B2PPQ is red shifted compared to that in both B1PPQ and BtBPQ. This is a direct consequence of elongation of the conjugation length. ${ }^{61}$ This shift is not very significant in calculations and is less pronounced in experiment due to torsional distortion between aryls at the ends, which disrupts conjugation. Nevertheless, the terminal aryls are participating in this excitation (see Table 8). In contrast, the aryl substitutions in BDBPQ do not have any substantial effect on the lowest-state excitation energy, which is closest to that in BtBPQ, compared to the other three members of the family. It is well established that the electronic delocalization through the meta-position in the phenyl ring substitutions is effectively blocked, ${ }^{62}$ and such substitutions do not usually bear significant effects. Consequently, the electronic state does not delocalize on the four terminal phenyls, as illustrated by the respective transition orbitals (see Table 8). In fact, the relative ordering of the energy of the first excited state observed in experiment ${ }^{1}$ (from blue to red, B1PPQ, BDBPQ, BtBPQ, $\mathrm{B} 2 \mathrm{PPQ}$ ) is well reproduced by all computational methods (Table 16). Compared to B1PPQ, we further observe that, due to the extended conjugation, the value of the oscillator strength increases substantially in B2BPQ. This effect is smaller in BDBPQ.

The energetics of the two higher-lying excited states contributing to the second absorption peak is substantially changed due to aryl substitutions in B2PPQ and BDBPQ. Noteworthy, these two excitations are even more multiconfigurational. For 
TABLE 7: TDDFT Natural Transition Orbital Analysis for the Three Excited States with the Largest Oscillator Strengths in B2PPQ in the Gas Phase ${ }^{a}$

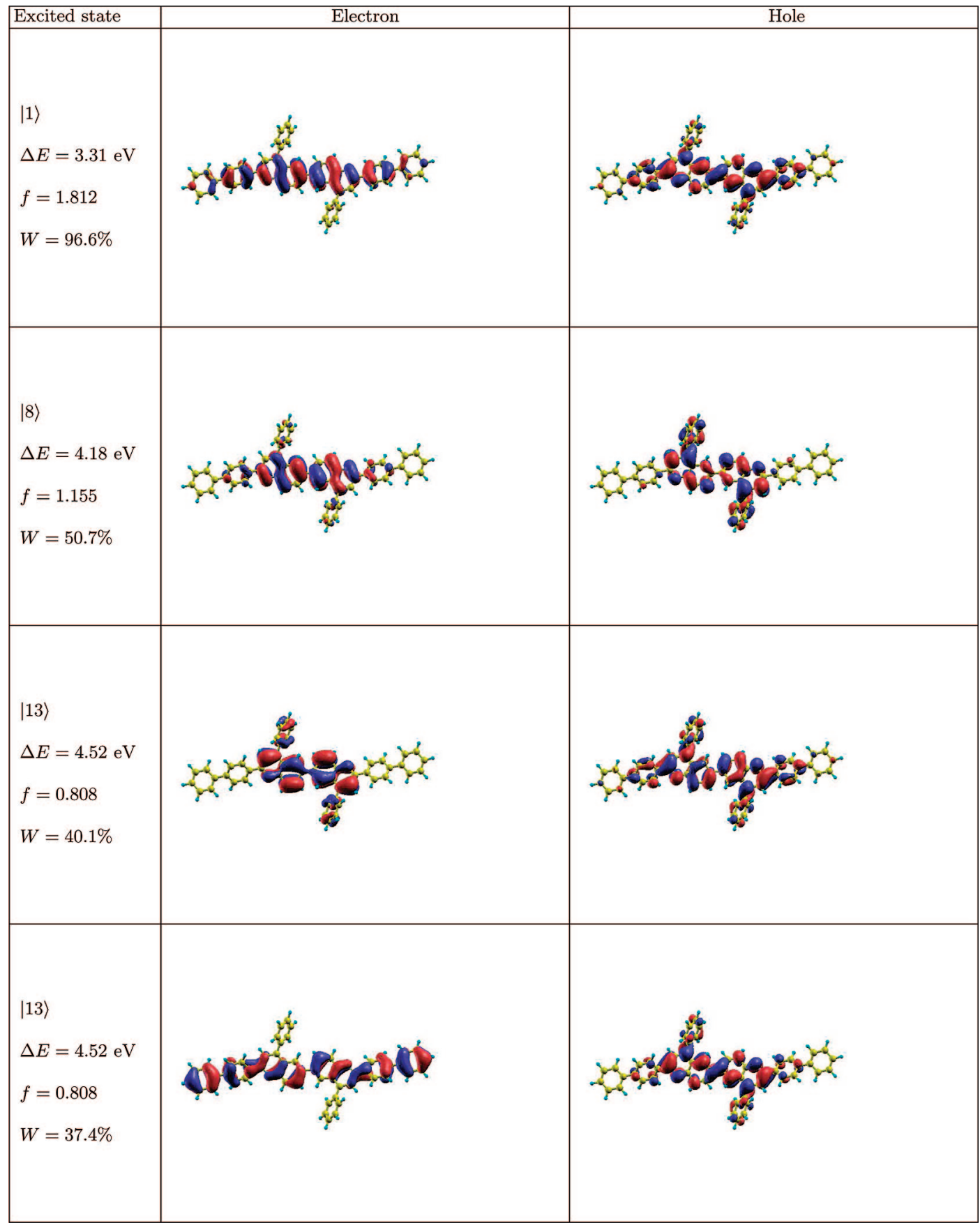

${ }^{a} \Delta E$ is the excitation energy, $f$ is the corresponding oscillator strength, and $W$ is the weight of the plotted orbital in the respective transition density matrix.

example, excited state $|13\rangle$ in B2PPQ is a mixture of two transitions between pairs of transition orbitals; the first pair corresponds to the transition in the middle section of the molecules, involving the "middle" aryls, whereas the second pair describes charge transfer from the terminal phenyls to the center (see Table 7). Roughly a similar picture holds for excited state $|10\rangle$ in BDBPQ (see Table 7). Such high-energy excited states can delocalize through the barrier imposed by metapositions on the molecular structure. Indeed, both contributions to excited state $|13\rangle$ in BDBPQ represent partial charge redistribution from the terminal phenyls to the center of the oligomer (only one contribution is shown in Table 8).
Spectroscopically, we observe splitting of the second peak in the simulated absorption spectrum of B2PPQ (see Figure 4), where both maxima have smaller magnitudes compared to the first absorption peak. Experimentally, only one absorption peak is observed in the higher-frequency region in B2PPQ (not shown). However, its intensity is substantially lower compared to the other molecules in the family. Among other computed properties, the energies of the lowest triplet state are the same in three molecules (B1PPQ, BtBPQ, and BDBPQ) and show only a moderate red shift due to the extended conjugation length in B2PPQ (see Tables 1-4). Triplet states also display minimal solvatochromic shifts (about $10 \mathrm{meV}$ ) and typically have a very 
TABLE 8: TDDFT Natural Transition Orbital Analysis for the Three Excited States with the Largest Oscillator Strengths in BDBPQ in the Gas Phase ${ }^{a}$

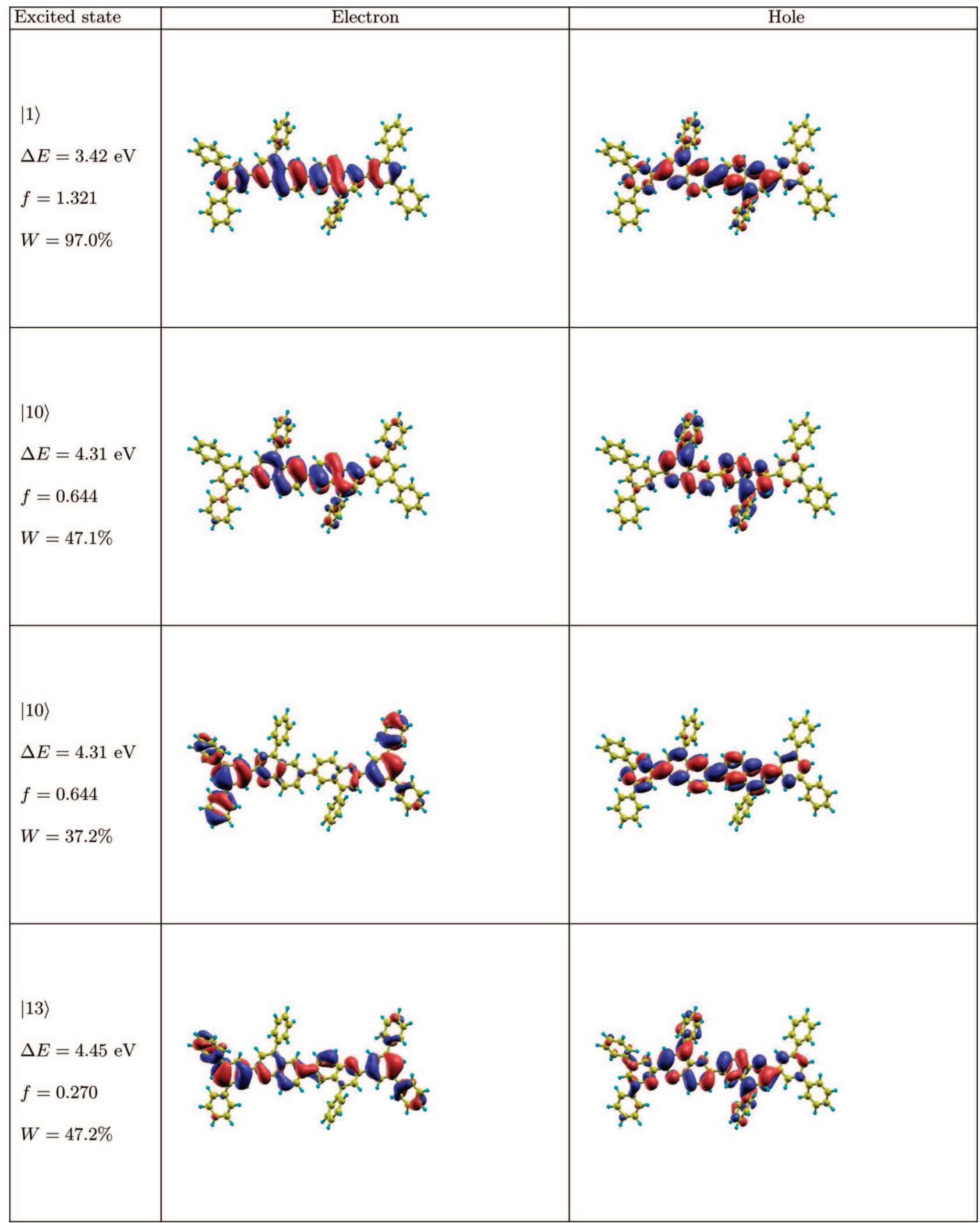

${ }^{a} \Delta E$ is the excitation energy, $f$ is the corresponding oscillator strength, and $W$ is the weight of the plotted orbital in the respective transition density matrix.

localized nature. ${ }^{34}$ Note that the ground-state dipole moment (which is approximately directed orthogonally to the molecular backbone) is roughly the same in all four molecules investigated.

\section{Conclusion}

In the present work, we have employed time-dependent density functional theory with the adiabatic approximation for the dynamical XC potential, which is constructed from a ladder of density functionals used in the routine static DFT calculations, to investigate the absorption spectra of blue-light-emitting oligoquinolines (B1PPQ, BtBPQ, B2PPQ, BDBPQ) in the gas phase and in chloroform solution. Our calculations are in good agreement with the experimental measurements of absorption spectra in chloroform solution.

Our results show that there are two absorption peaks for B1PPQ, BDBPQ, and BtBPQ molecules in the gas phase and solution, while an additional higher-frequency absorption peak may occur for the B2PPQ molecule. The first absorption peak arises from the lowest singlet-singlet transition, whereas the second arises from multiple excited states. We simulate the experimental spectra with the data calculated from the adiabatic PBE0 density functional using an analytic one-parameter formula and find that our simulation agrees well with the experimentally measured absorptions. To spell out the underly- 
ing mechanism for these excitations, an analysis of the natural transition orbitals corresponding to the excited states in question has been made. We find that for the whole family of oligoquinoline molecules, the lowest excited state is formed by a delocalized excitation involving the entire conjugated backbone, while the higher excited states are formed by less delocalized excitations.

Compared to the absorptions in the gas phase, there is a consistent relative red shift in the absorption wavelength. Furthermore, because of the solvent-solute interaction, the dipole moment and thus the oscillator strength are larger in solution than in the gas phase.

It is worth noting that the order of accuracy of the five adiabatic density functionals in predicting the low-lying excitation energies of small molecules, as found in our previous study, ${ }^{18}$ that is

$$
\text { LSDA }<\text { TPSS }<\text { TPSSh }<\text { B3LYP }<\text { PBE0 }
$$

continues to hold even for large molecules, as shown in Tables $1-4$.

Acknowledgment. We thank Ekaterina Badaeva for technical help and Svetlana Kilina for useful discussions. This work was carried out under the auspices of the National Nuclear Security Administration of the U.S. Department of Energy at Los Alamos National Laboratory under Contract No. DE-AC52-06NA25396 and under the Grant No. LDRD-PRD X9KU.

\section{References and Notes}

(1) Tonzola, C. J.; Kulkarni, A. P.; Gifford, A. P.; Kaminsky, W.; Jenekhe, S. A. Adv. Funct. Mater. 2007, 17, 863.

(2) Forrest, S. R. Nature 2004, 428, 911.

(3) Kraft, A.; Grimsdale, A. C.; Holmes, A. B. Angew. Chem., Int. Ed. 1998, 37, 402

(4) Mitschke, U.; Bauerle, P. J. J. Mater. Chem. 2000, 10, 1471 1089 .

(5) Kim, D. Y.; Cho, H. N.; Kim, C. Y. Prog. Polym. Sci. 2000, 25 ,

(6) Kulkarni, A. P.; Tonzola, C. J.; Babel, A.; Jenekhe, S. A. Chem. Mater. 2004, 16, 4556.

(7) Heeger, A. J.; Heeger, D. J.; Langan, J.; Yang, Y. Science 1995, 270,1642

(8) Sirringhaus, H.; Kawase, T.; Friend, R. H.; Shimoda, T.; Inbasekaran, M.; Wu, W.; Woo, E. P. Science 2000, 290, 2123.

(9) Menard, E.; Meitl, M.; Sun, Y.; Park, J.-U.; Shir, D.-L.; Nam, Y.S.; Jeon, S.; Rogers, J. Chem. Rev. 2007, 107, 1117.

(10) Granstrom, M.; Petritsch, K.; Arias, A. C.; Lux, A.; Andersson, M. R.; Friend, R. H. Nature 1998, 395, 257.

(11) Yu, G.; Gao, J.; Hummelen, J. C.; Wudl, F.; Heeger, A. J. Science 1995, 270, 1789.

(12) Sirringhaus, H.; et al. Nature 1999, 401, 685.

(13) Giuliani, G. F. ; Vignale, G. Quantum Theory of the Electron Liquid; Cambridge University Press: New York, March 2005.

(14) Time-Dependent Density Functional Theory, Lecture Notes in Physics; Marques, M. A. L., Ullrich, C. A., Nogueira, F., Rubio, A., Burke, K., Gross, E. K. U., Eds.; Springer: Berlin, Germany, 2006, Vol. 706.

(15) Kohn, W.; Sham, L. J. Phys. Rev. 1965, 140, A1133.

(16) Runge, E.; Gross, E. K. U. Phys. Rev. Lett. 1984, 52, 997.

(17) Petersilka, M.; Gossmann, U. J.; Gross, E. K. U. Phys. Rev. Lett. 1996, 76, 1212.

(18) Tao, J.; Tretiak, S.; Zhu, J.-X. J. Chem. Phys. 2008, 128, 084110

(19) Bauernschmitt, R.; Ahlrichs, R. Chem. Phys. Lett. 1996, $256,454$.

(20) Furche, F. J. Chem. Phys. 2001, 114, 5982.

(21) (a) Zangwill, A.; Soven, P. Phys. Rev. Lett. 1980, 45, 204. (b)

Zangwill, A.; Soven, P. Phys. Rev. A 1980, 21, 1561.

(22) Gross, E. K. U.; Kohn, W. Phys. Rev. Lett. 1985, 55, 2850.

(23) Vignale, G.; Kohn, W. Phys. Rev. Lett. 1996, 77, 2037.

(24) Tao, J.; Vignale, G. Phys. Rev. Lett. 2006, $97,036403$.

(25) Tao, J.; Vignale, G.; Tokatly, I. V. Phys. Rev. B 2007, 76, 195126.

(26) Ullrich, C. A.; Tokatly, I. V. Phys. Rev. B 2006, 73, 235102.

(27) Tokatly, I. V. Phys. Rev. B 2005, 71, 165105.

(28) Tozer, D. J.; Handy, N. C. J. Chem. Phys. 1998, 109, 10180.
(29) Stratmann, R. E.; Scuseria, G. E. J. Chem. Phys. 1998, 109, 8218. (30) Adamo, C.; Scuseria, G. E.; Barone, V. J. Chem. Phys. 1999, 111, 2889.

(31) Schipper, P. R. T.; Gritsenko, O. V.; van Gisbergen, S. J. A.; Baerends, E. J. J. Chem. Phys. 2000, 112, 1344.

(32) Sala, F. D.; Görling, A. Int. J. Quantum Chem. 2003, 91, 131.

(33) Jacquemin, D.; Bouhy, M.; Perpète, E. A. J. Chem. Phys. 2006, 124,204321

(34) Tretiak, S. Nano Lett. 2007, 7, 2201.

(35) Adams, R. D.; Captain, B.; Hall, M. B.; Trufan, E.; Yang, X. Z. J. Am. Chem. Soc. 2007, 129, 12328.

(36) Sancho-García, J. C. Chem. Phys. Lett. 2007, 439, 236.

(37) Igumenshchev, K. I.; Tretiak, S.; Chernyak, V. Y. J. Chem. Phys. 2007, 127, 114902.

(38) Perdew, J. P.; Burke, K.; Ernzerhof, M. Phys. Rev. Lett. 1996, 77, 3865.

(39) Tao, J.; Perdew, J. P.; Staroverov, V. N.; Scuseria, G. E. Phys. Rev. Lett. 2003, 91, 146401.

(40) Perdew, J. P.; Tao, J.; Staroverov, V. N.; Scuseria, G. E. J. Chem. Phys. 2004, 120, 6898.

(41) Staroverov, V. N.; Scuseria, G. E.; Tao, J.; Perdew, J. P. J. Chem. Phys. 2003, 119, 12129.

(42) Csonka, G. I.; Ruzsinszky, A.; Tao, J.; Perdew, J. P. Int. J. Quantum Chem. 2005, 101, 506.

(43) Furche, F.; Perdew, J. P. J. Chem. Phys. 2006, 124, 044103.

(44) Staroverov, V. N.; Scuseria, G. E.; Tao, J.; Perdew, J. P. Phys. Rev. B 2004, 69, 075102.

(45) Tao, J.; Perdew, J. P. Phy. Rev. Lett. 2005, 95, 196403.

(46) Tao, J.; Vignale, G.; Tokatly, I. V. Phys. Rev. Lett. 2008, 100, 206405.

(47) Stephens, P. J.; Devlin, F. J.; Chabalowski, C. F.; Frisch, M. J. J. Phys. Chem. 1994, 98, 11623.

(48) Martin, R. L. J. Chem. Phys. 2003, 118, 4775.

(49) Frisch, M. J. Trucks, G. W.; Schlegel, H. B.; Scuseria, G. E.; Robb, M. A.; Cheeseman, J. R.; Montgomery, J. A., Jr.; Vreven, T.; Kudin, K. N.; Burant, J. C.; Millam, J. M.; Iyengar, S. S.; Tomasi, J.; Barone, V.; Mennucci, B.; Cossi, M.; Scalmani, G.; Rega, N.; Petersson, G. A.; Nakatsuji, H.; Hada, M.; Ehara, M.; Toyota, K.; Fukuda, R.; Hasegawa, J.; Ishida, M.; Nakajima, T.; Honda, Y.; Kitao, O.; Nakai, H.; Klene, M.; Li, X.; Knox, J. E.; Hratchian, H. P.; Cross, J. B.; Bakken, V.; Adamo, C.; Jaramillo, J.; Gomperts, R.; Stratmann, R. E.; Yazyev, O.; Austin, A. J.; Cammi, R.; Pomelli, C.; Ochterski, J. W.; Ayala, P. Y.; Morokuma, K.; Voth, G. A.; Salvador, P.; Dannenberg, J. J.; Zakrzewski, V. G.; Dapprich, S.; Daniels, A. D.; Strain, M. C.; Farkas, O.; Malick, D. K.; Rabuck, A. D.; Raghavachari, K.; Foresman, J. B.; Ortiz, J. V.; Cui, Q.; Baboul, A. G.; Clifford, S.; Cioslowski, J.; Stefanov, B. B.; Liu, G.; Liashenko, A.; Piskorz, P.; Komaromi, I.; Martin, R. L.; Fox, D. J.; Keith, T.; Al-Laham, M. A.; Peng, C. Y.; Nanayakkara, A.; Challacombe, M.; Gill, P. M. W.; Johnson, B.; Chen, W.; Wong, M. W.; Gonzalez, C.; Pople, J. A. Gaussian 03; Gaussian, Inc.: Pittsburgh, PA, 2003.

(50) Curtiss, L. A.; Redfern, P. C.; Raghavachari, K.; Pople, J. A. J. Chem. Phys. 2001, 114, 108.

(51) Perdew, J. P.; Ruzsinszky, A.; Tao, J.; Csonka, G. I.; Scuseria, G. E. Phys. Rev. A 2007, 76, 042506.

(52) Perdew, J. P.; Wang, Y. Phys. Rev. B 1992, 45, 13244.

(53) Cancès, E.; Mennucci, B.; Tomasi, J. J. Chem. Phys. 1997, 107, 3032.

(54) Cossi, M. Chem. Phys. Lett. 2003, 384, 179.

(55) Cossi, M.; Rega, N.; Scalmani, G.; Barone, V. J. Comput. Chem.

2003, 24, 669 .

(56) Arfken, G. Mathematical Methods for Physicists, 3rd ed.; Academic Press: New York, 1985.

(57) Chicanne, C.; David, T.; Quidant, R.; Weeber, J. C.; Lacroute, Y.; Bourillot, E.; Dereux, A.; Colas des Francs, G.; Girard, C. Phys. Rev. Lett. 2002, 89, 097402.

(58) Kasha, M. Discuss Faraday Soc. 1950, 9, 14

(59) Wilson, J. S.; Dhoot, A. S.; Seeley, A. J. A. B.; Khan, M. S.; Köhler, A.; Friend, R. H. Nature 2001, 413, 828.

(60) (a) Monkman, A. P.; Burrows, H. D.; Hartwell, L. J.; Horsburgh, L. E.; Hamblett, I.; Navaratnam, S. Phys. Rev. Lett. 2001, 86, 1358. (b) Burrows, H. D.; Seixas de Melo, J.; Serpa, C.; Arnaut, L. G.; Miguel, M. da G.; Monkman, A. P.; Hamblett, I.; Navaratnam, S. Chem. Phys. 2002, 285,3 .

(61) Tretiak, S.; Mukamel, S. Chem. Rev. 2002, 102, 3171.

(62) (a) Tretiak, S.; Chernyak, V.; Mukamel, S. J. Phys. Chem. B 1998, 102, 3310. (b) Wu, C.; Malinin, S. V.; Tretiak, S.; Chernyak, V. Y. Nat. Phys. 2006, 2, 631. (c) Kopelman, R.; Shortreed, M.; Shi, Z.-Y.; Tan, W.; Xu, Z.; Moore, J. S.; Bar-Haim, A.; Klafter, J. Phys. Rev. Lett. 1997, 78, 1239.

\section{JP804687J}

